Journal of Bangladesh Academy of Sciences, Vol. 34, No. 2, 143-152, 2010

\title{
DISCRETIZED CURRENT DISTRIBUTION FOR ACTIVE ENDCAP TRANSVERSE GRADIENT COIL DESIGN FOR MAGNETIC RESONANCE IMAGING USING STREAM FUNCTION APPROACH
}

\author{
G. DASTEGIR AL-QUADERI ${ }^{1}$, M. ARSHAD MOMEN ${ }^{2}$ AND TANVIR NOOR BAIG $^{3}$ \\ Department of Physics, University of Dhaka, Dhaka-1000, Bangladesh
}

\begin{abstract}
The authors present the discretized form of the current distribution using the stream function approach starting from a continuous current distribution obtained recently for an active endcap transverse gradient coil in Magnetic Resonance Imaging (MRI) machine. The present discretized current distribution is compared with the one found using apodized current distributions on the cylinder and the cap surfaces separately and its advantages are discussed.
\end{abstract}

Key words: Gradient coils, Stream function; Active endcap

\section{INTRODUCTION}

Gradient coils in a Magnetic Resonance Imaging (MRI) machine are used to generate linearly varying magnetic fields along the $x-, y$ - and $z$-axes. They give a unique magnetic field at each point within the region of interest (FOV) resulting in a unique frequency of the signal from the resonance process. These coils are turned off and on rapidly during a scanning sequence producing eddy current effects on external metallic structures such as the magnet cold shields, the magnet dewar, and the like. To resolve this problem a second set of coils, placed between the primary gradient coil and the effected structures are introduced, known as the shielding coils. However, the conventional design of cylindrical shaped secondary shielding coils can not stem the fringe field outside completely. Baig et al. (2007) proposed a new way to design shielded gradient coils by augmenting the current density of the shielding coil on the surface of a cylinder by an endcap distribution that has azimuthal and radial current distributions. The design produced reduction on the fringe field compared with a traditional shielded gradient coil design and that, in turn, showed a reduction in acoustic noise on the external metallic structures. However, the design presented by Baig et al. (2007) were not optimized in terms of continuous current density distribution on the combined surface of the secondary. The continuity of the current distribution between the cylindrical part and the endcap parts were imposed after the discretization of the coil was done. This resulted in significant loss of shielding ability of this new gradient coil design.

\footnotetext{
${ }^{1}$ Corresponding author: < dastegir@gmail.com>., 2. <amomen@univdhaka.edu>.

${ }^{3}<$ tnbaig@yahoo.com>.
} 
In a recent work Al-Quaderi et al. (2010) solved for the continuous current distribution on the combined surface of the cylindrical secondary and the endcap surfaces. The current distribution found by them were general enough to include only the cylindrical secondary or the solely the endcap shielding coils. It also allowed the endcaps to be tilted at an angle $\alpha$ generalizing from the case of perpendicular endcaps treated in Baig et al. (2007). The present paper uses the stream function approach to discretize the proposed current density in Al-Quaderi et al. (2010). The stream function is defined and found for the cylindrical and the endcap surfaces. The continuity of the current is automatically satisfied as the explicit form of the stream functions are constructed. The difference of the values of the stream function between two streamlines gives the current contained within the lines. The authors plot the streamlines and get the discretized current paths. At the end of the article the authors discuss their results comparing with the results of other authors and the possible implications of these in the design of MRI coils.

\section{STREAM FUNCTION FOR THE CURRENT DENSITIES}

The authors consider a cylindrical secondary surface and two caps attached at its two ends. The thickness of the coils are taken to be infinitesimal for the sake of simplicity making the current distribution confined on two-dimensional surfaces. The endcaps are tilted at an angle $\alpha$ with the cylinder's axis. The confinement of the current density is implemented by using the Dirac delta function and the Heaviside step function as:

$$
\begin{aligned}
& J_{\rho, \phi, z}^{(C)}(\vec{r}) \sim K_{\rho, \phi, z}^{(C)} \delta(\mathrm{z} \pm \mathrm{Z}(\rho)) \Theta\left(\rho-\rho_{0}\right) \Theta\left(R_{S}-\rho\right) \\
& J_{\phi, z}^{(S)}(\vec{r}) \sim K_{\phi, z}^{(S)} \delta\left(\rho-R_{S}\right) \Theta\left(\mathrm{z}+L_{S}\right) \Theta\left(L_{S}-z\right)
\end{aligned}
$$

where the minus/plus sign corresponds to the caps that ends at $z= \pm L_{S}$, respectively. Here $L_{S}$ is the half-length of the cylindrical secondary surface, $\rho_{0}$ is the radius of the inner end of the caps and $R_{S}$ is that of the secondary cylindrical surface, respectively and $K_{\rho, \phi, z}$ are the components of the surface current density. The 1D delta function has the dimension of inverse of length and hence $[K]=[I / L]$.

The equation of the cylindrical surface is $\rho-R_{S}=0$ while that of the caps ending at $z= \pm L_{S}$ are

$$
z= \pm Z(\rho)= \pm\left[L_{\text {in }}+\left(\rho-\rho_{0}\right) \cot \alpha\right]
$$

where $L_{\text {in }}$ is the distance of the inner end of the caps from $z=0$ along the $z$-direction on the two sides and $\cot \alpha=\left(L_{S}-L_{\text {in }}\right) /\left(R_{S}-\rho_{0}\right)$. 


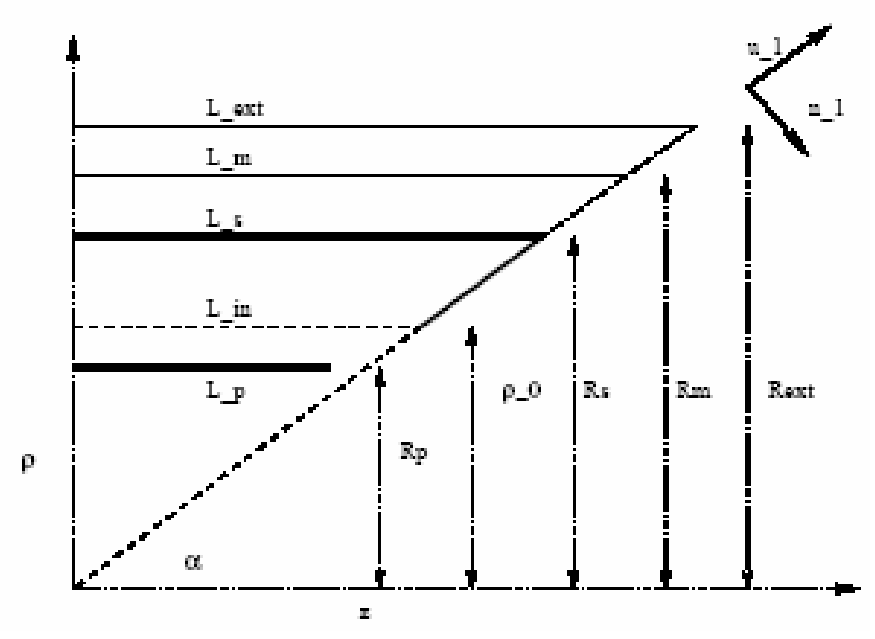

Fig. 1. Geometry of the X-gradient coil with tilted end-caps shown schematically for half of the length of the coils. Here $\left(L_{\mathrm{P}}, R_{\mathrm{P}}\right),\left(L_{\mathrm{S}}, R_{\mathrm{S}}\right),\left(L_{\mathrm{m}}, R_{\mathrm{m}}\right)$ and $\left(L_{\mathrm{ext}}, R_{\text {ext }}\right)$ are the halflengths and the radii of the primary, secondary, main magnet and the external shielding surfaces, respectively.

The stream function: The continuity equation for the current density $\vec{J}(\vec{r})$ reduces to the zero-divergence equation $\vec{\nabla} \cdot \vec{J}=0$ in the absence of any accumulation or depletion of charge density. In cylindrical coordinates

$$
\frac{1}{\rho}\left[\frac{\partial}{\partial \rho}\left(\rho J_{\rho}\right)+\frac{\partial J_{\phi}}{\partial \phi}+\rho \frac{\partial J_{z}}{\partial z}\right]=0 \quad \Rightarrow \quad \vec{\nabla} \cdot \vec{K}=0
$$

for all points on the surface. Hence, authors can express the surface current density as a curl of some vector field $\vec{S}$ :

$$
\vec{K}=\vec{\nabla} \times \vec{S}
$$

Since, $\vec{K}$ exists only on the surface, the vector field $\vec{S}$ has component perpendicular to the surface as well. The rotation or curl of $\vec{S}$ will point tangential to the surface.

Definition: If a continuous surface current density $\vec{K}$ can be expressed as the curl of a vector field, which has non-zero component only normal to the surface, then that component is called the stream function of the surface current density on the particular surface.

Hence, the stream function $S$ is related to $\vec{S}$ by $\vec{S}=(\vec{S} . \hat{n}) \hat{n}=S \hat{n}$, where $\hat{n}$ is a normal vector to the surface.

Stream function on the cylindrical surface: The general form of the surface current density components on the cylindrical surface can be found from symmetry considerations as in Al-Quaderi et al. (2010) 


$$
\begin{aligned}
& K_{\phi}^{(S)}=f_{\phi}^{(S)}(z) \Theta\left(z+L_{S}\right) \Theta\left(L_{S}-z\right) \cos (\phi) \\
& K_{z}^{(S)}=f_{z}^{(S)}(z) \Theta\left(z+L_{S}\right) \Theta\left(L_{S}-z\right) \sin (\phi)
\end{aligned}
$$

where $f_{\phi}^{(S)}(z)$ and $f_{z}^{(S)}(z)$ are coefficient functions for the surface current densities.

Consider a continuous surface current density $\vec{K}^{(S)}=\vec{K}^{(S)}(\phi, z)$ confined on the cylindrical Surface $\rho=R_{S}$. We can express $\vec{K}^{(S)}=K_{\phi}^{(S)} \hat{e}_{\phi}+K_{z}^{(S)} \hat{e}_{z}$, such that $\vec{K}=\vec{\nabla} \times \vec{S}$. The equation of continuity for $\vec{K}^{(S)}$ reduces to

$$
\frac{\partial}{\partial \phi} K_{\phi}^{(S)}+\rho \frac{\partial}{\partial z} K_{z}^{(S)}=0
$$

The stream function $S$ is the radial component of a vector field $\vec{S}=\left(S^{(S)}(\phi, z), 0,0\right)$. Plugging this into the curl definition, the authors get,

$$
\begin{gathered}
K_{\phi}^{(S)}=\frac{\partial S^{(S)}}{\partial z} \\
K_{z}^{(S)}=-\frac{1}{R_{S}} \frac{\partial S^{(S)}}{\partial \phi}
\end{gathered}
$$

Upon integration of (9), (10), the authors get two forms of $S^{(S)}$ as:

$$
\begin{aligned}
S^{(S)} & =\left[\int f_{\phi}^{(S)}(z) \Theta\left(z+L_{S}\right) \Theta\left(L_{S}-z\right) d z\right] \cos (\phi)+F_{1}(\phi) \\
& =R_{S} f_{z}^{(S)}(z) \Theta\left(z+L_{S}\right) \Theta\left(L_{S}-z\right) \cos (\phi)+G_{1}(\mathrm{z})
\end{aligned}
$$

where, $F_{1}(\phi)$ and $G_{1}(z)$ are arbitrary functions. Putting $\phi=\pi / 2$, the authors get $F_{1}(\pi / 2)=G_{1}(z)$ i.e. $G_{1}(z)$ is a constant function. Ignoring the unimportant constant, we get

$$
\begin{aligned}
S^{(S)} & =\left[\int f_{\phi}^{(S)}(z) \Theta\left(z+L_{S}\right) \Theta\left(L_{S}-z\right) d z\right] \cos (\phi) \\
& =R_{S} f_{z}^{(S)}(z) \Theta\left(z+L_{S}\right) \Theta\left(L_{S}-z\right) \cos (\phi)
\end{aligned}
$$

Putting in the Fourier expansion of $f_{z}^{(S)}(z)$ or of $f_{\phi}^{(S)}(z)$ from Al-Quaderi et al. (2010), the authors get

$$
S^{(S)}\left(\rho=R_{S}, \phi, z\right)=R_{S}\left[\sum_{n} a_{n}^{(S)} \sin \left(\frac{\pi n z}{\widetilde{L}_{S}}\right)\right] \Theta\left(z+L_{S}\right) \Theta\left(L_{S}-z\right) \cos (\phi)
$$

where $\tilde{L}_{S}=L_{S}+\left(R_{S}-\rho_{0}\right)$. Note that, at the edge of the cylinder, we have, $S^{(S)}\left(\rho=R_{S}\right.$, $\left.\phi, \quad z= \pm L_{S}\right)=R_{S} f_{z}^{(S)}\left( \pm L_{S}\right) \cos (\phi) \neq 0$, in general. 
Stream function on the tilted endcaps: On the tilted end caps, the volume current densities are confined on the cap surfaces and hence

$$
\vec{J}_{1,2}^{(C)}=\delta(z \mp Z(\rho)) \vec{K}_{1,2}^{(C)}
$$

where $z= \pm Z(\rho)$ are the equations of the cap surfaces. Since the caps are tilted in an arbitrary angle $\alpha, \vec{K}^{(C)}$ has in general, all three i.e. $\rho, \phi, z$-components. The components $K_{1,2} \rho, \phi$, are functions of $(\rho, \phi)$ only, since the $z$-dependence may be eliminated by the equations of the surfaces $z= \pm Z(\rho)$. Hence the authors get

$$
\vec{K}_{1,2}^{(C)}=\vec{K}_{1,2}^{(C)}(\rho, \phi)=K_{\rho 1,2}^{(C)} \hat{e}_{\rho}+K_{\phi 1,2}^{(C)} \hat{e}_{\phi}+K_{z 1,2}^{(C)} \hat{e}_{z}
$$

From the equation of continuity $\vec{\nabla} \cdot \vec{J}=0$, the authors get

$$
\begin{aligned}
& \delta(z \mp Z(\rho))\left[\frac{\partial}{\partial \rho}\left(\rho K_{\rho 1,2}^{(C)}\right)+\frac{\partial}{\partial \phi} K_{\phi 1,2}^{(C)}\right] \\
& +\rho \delta^{\prime}(z \mp Z(\rho))\left[\mp \cot (\alpha) K_{\rho 1,2}^{(C)}+K_{z 1,2}^{(C)}\right]=0
\end{aligned}
$$

which gives, using the linear independence of Dirac delta function and its derivative,

$$
\begin{aligned}
& \frac{\partial}{\partial \phi} K_{\phi 1,2}^{(C)}=-\frac{\partial}{\partial \rho}\left(\rho K_{\rho 1,2}^{(C)}\right) \\
& K_{z 1,2}^{(C)}= \pm \cot (\alpha) K_{\rho 1,2}^{(C)}
\end{aligned}
$$

The unit vectors perpendicular to the endcaps are $\hat{n}_{1,2}= \pm \sin (\alpha) \hat{e}_{z}-\cos (\alpha) \hat{e}_{\rho}$. The component of $\vec{K}$ along $\hat{n}_{1,2}$ are

$$
\begin{aligned}
& K_{\perp 1}^{(C)}=\vec{K}_{1}^{(C)} \cdot \hat{n}_{1}=\sin (\alpha) K_{z 1}^{(C)}-\cos (\alpha) K_{\rho 1}^{(C)} \\
& K_{\perp 2}^{(C)}=\vec{K}_{2}^{(C)} \cdot \hat{n}_{2}=\sin (\alpha) K_{z 2}^{(C)}+\cos (\alpha) K_{\rho 1}^{(C)}
\end{aligned}
$$

which are zero using (20), as expected.

The continuous surface current densities can be expressed as curls of some vector fields:

$$
\vec{K}_{1,2}^{(C)}=\vec{\nabla} \times \vec{S}_{1,2}
$$

The authors assume that the only non-zero components of $\vec{S}_{1,2}$ are the ones perpendicular to the endcap surfaces and the tangential components are zero. This can always be done as there is a gauge freedom for choosing the components of $\vec{S}$ as $\vec{S} \rightarrow \vec{S}+\vec{\nabla} \chi$ and hence the authors have

$$
S_{\phi}=0, \quad S_{u 1,2}= \pm S_{z 1,2} \cos (\alpha)+S_{\rho 1,2} \sin (\alpha)=0
$$


which gives

$$
\vec{S}_{1,2}=\left(S_{\rho 1,2}, 0, S_{z 1,2}\right)=(\mp \cot (\alpha), 0,1) S_{z 1,2}
$$

The stream functions $\Psi_{1,2}$ are the normal components of $\vec{S}_{1,2}$ on the tilted endcaps:

$$
\Psi_{1,2}=\hat{n}_{1,2} \cdot \vec{S}_{1,2}= \pm \frac{1}{\sin (\alpha)} S_{z 1,2}
$$

The unit vectors tangential to the cap-surfaces are $\hat{e}_{\phi}$ and $\hat{u}_{1,2}= \pm$ $\cos (\alpha) \hat{e}_{z}+\sin (\alpha) \hat{e}_{\rho}$. Define two variables, $u_{1,2}$ which increase along the tilted endcaps in the outward directions:

$$
u_{1,2}=\vec{r} \cdot \hat{u}_{1,2}=\rho \sin (\alpha) \pm z \cos (\alpha)
$$

Then, the directional derivatives along increasing $u_{1,2}$ are:

$$
\frac{\partial}{\partial u_{1,2}}=\hat{u}_{1,2} \cdot \vec{\nabla} \equiv \sin (\alpha) \frac{\partial}{\partial \rho} \pm \cos (\alpha) \frac{\partial}{\partial z}
$$

Hence, from the curl relation, the authors get

$$
\begin{aligned}
K_{\rho 1,2}^{(C)}(\rho, \phi)= & \frac{1}{\rho} \frac{\partial}{\partial \phi} S_{z 1,2}= \pm \frac{\sin (\alpha)}{\rho} \frac{\partial}{\partial \phi} \Psi_{1,2} \\
K_{\phi 1,2}^{(C)}(\rho, \phi)= & \frac{\partial}{\partial z} S_{\rho 1,2}-\frac{\partial}{\partial \rho} S_{z 1,2} \\
& =\left[\mp \sin (\alpha) \frac{\partial}{\partial \rho}-\cos (\alpha) \frac{\partial}{\partial z}\right] \Psi_{1,2} \\
& =\mp \frac{\partial}{\partial u_{1,2}} \Psi_{1,2} \\
& K_{z 1,2}^{(C)}(\rho, \phi)=-\frac{1}{\rho} \frac{\partial}{\partial \phi} S_{\rho 1,2}=\frac{\cos (\alpha)}{\rho} \frac{\partial}{\partial \phi} \Psi_{1,2}
\end{aligned}
$$

The general form of the surface current density components on the endcaps can be assumed from the symmetry considerations as in Al-Quaderi et al. (2010) as

$$
\begin{aligned}
& K_{\rho 1,2}^{(C)}(\rho, \phi)=\mathrm{g}_{\rho}^{(C)}(\rho) \Theta\left(\rho-\rho_{0}\right) \Theta\left(R_{S}-\rho\right) \sin (\phi) \\
& K_{\phi 1,2}^{(C)}(\rho, \phi)=\mathrm{g}_{\phi}^{(C)}(\rho) \Theta\left(\rho-\rho_{0}\right) \Theta\left(R_{S}-\rho\right) \cos (\phi) \\
& K_{z 1,2}^{(C)}(\rho, \phi)= \pm \mathrm{g}_{z}^{(C)}(\rho) \Theta\left(\rho-\rho_{0}\right) \Theta\left(R_{S}-\rho\right) \sin (\phi)
\end{aligned}
$$


Putting the above into the stream function relations (29), (32) and upon integration, the authors get

$$
\begin{aligned}
& \Psi_{1,2}=\mp \frac{\rho}{\sin (\alpha)} \mathrm{g}_{\rho}^{(\mathrm{C})}(\rho) \Theta\left(\rho-\rho_{0}\right) \Theta\left(R_{S}-\rho\right) \cos (\phi)+P(\rho, z) \\
& \Psi_{1,2}=\mp \frac{\rho}{\cos (\alpha)} \mathrm{g}_{z}^{(\mathrm{C})}(\rho) \Theta\left(\rho-\rho_{0}\right) \Theta\left(R_{S}-\rho\right) \cos (\phi)+Q(\rho, z)
\end{aligned}
$$

Putting, $\phi=\pi / 2$, we get, $P(\rho, z)=Q(\rho, z)$, i.e. $P$ and are the same functions. Hence, the authors get, $g_{z}^{(C)}(\rho)=\cot (\alpha) g_{\rho}^{(C)}(\rho)$, same as found before.

Putting in the Fourier expansion of $g_{\rho}^{(C)}(\rho)$ (or $g_{z}^{(C)}(\rho)=\cot (\alpha) g_{\rho}^{(C)}(\rho)$ ) from AlQuaderi et. al. (2010), the authors get

$$
\Psi_{1,2}=\mp \frac{1}{\sin (\alpha)}\left\{\sum_{n} t_{n}^{(C)} \sin \left[\frac{\pi n}{\widetilde{R}_{S}}\left(\rho-\rho_{0}\right)\right]\right\} \Theta\left(\rho-\rho_{0}\right) \Theta\left(R_{S}-\rho\right) \cos (\phi)+P(\rho, z)
$$

where $\widetilde{R}_{S}=L_{S}+\left(R_{S}-\rho_{0}\right)$. Again, from the relation (31), the authors get

$$
\Psi_{1,2}=\mp \int K_{\phi 1,2}^{(C)}(\rho, \phi) d u_{1,2}+R\left(t_{1,2}, \phi\right)
$$

where $t_{1,2}$ are distances along the normal direction to the endcaps. On the endcaps, $d z= \pm d \rho \cot (\alpha)$ and hence, $d u_{1,2}=\sin (\alpha) d \rho+\left(\cos ^{2}(\alpha) / \sin (\alpha)\right) d \rho=(d \rho / \sin (\alpha))$. Hence, upon integration,

$$
\Psi_{1,2}=\mp \frac{1}{\sin (\alpha)}\left\{\sum_{n} t_{n}^{(C)} \sin \left[\frac{\pi n}{\widetilde{R}_{S}}\left(\rho-\rho_{0}\right)\right]\right\} \Theta\left(\rho-\rho_{0}\right) \Theta\left(R_{S}-\rho\right) \cos (\phi)+R\left(t_{1,2}, \phi\right)
$$

Hence, comparing the authors get, $P(\rho, z)=R\left(t_{1,2}, \phi\right)$. Thus, $R\left(t_{1,2}, \phi\right)$ is a constant function of $\phi$. Since, $t_{1,2}=-\rho \cos (\alpha) \pm z \sin (\alpha)$, we can express $R\left(t_{1,2}\right)$ as a function of $\rho$ and $z$. For the perpendicular endcaps $\alpha=\pi / 2, u_{1,2}=\rho$ and $t_{1,2}= \pm z$. Considering the case of the perpendicular endcaps, the authors can show that $P(\rho, z)=0$. Hence, the stream functions on the tilted endcaps are:

$$
\Psi_{1,2}=\mp \frac{\rho}{\sin (\alpha)} g_{\rho}^{(C)}(\rho) \Theta\left(\rho-\rho_{0}\right) \Theta\left(R_{S}-\rho\right) \cos (\phi)
$$

Again, note that, at the edge of the cylinder, we have,

$$
\Psi_{1,2}\left(R_{S}, \phi, \pm L_{S}\right)=\mp \frac{R_{S}}{\sin (\alpha)} g_{\rho}^{(C)}\left(R_{S}\right) \quad \cos (\phi) \neq 0, \text { in general. }
$$




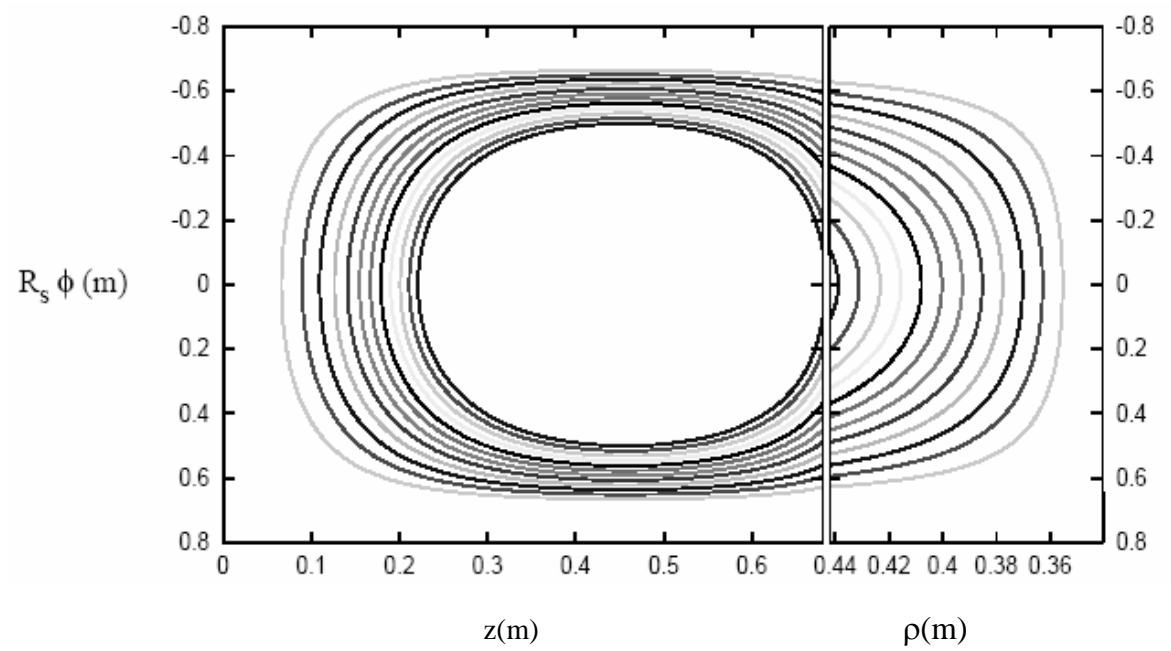

Fig. 2. The contour plots of the stream function on the cylindrical surface and the endcap at $z=L_{S}$. The values are from 400 to 2600 Amp with the increment of $200 \mathrm{Amp}$ for the set of coefficients chosen. The contours that end at the common edge on the cylinder meet at the same position $R_{S} \phi$ with the corresponding contour on the cap.

\section{GENERATING WINDING PATTERNS USING THE STREAM FUNCTION METHOD}

It can be shown that (Brideson et al. (2002), Peeren (2003)), any two streamlines having a fixed amount of difference between the stream functions on them will contain equal amount of current between them. Thus, if the want to get the discretized paths along which a constant current flows, the authors need to find the contours of the stream function on the surfaces. Thus, the authors consider streamlines, on either the cylindrical surface or the endcaps, having values of the stream functions differing by some specified amount, equal to the intended current that the authors want to flow through the discretely placed wires.

Let the maximum value of the stream function on a surface be $S_{\max }=I_{\max }$, the maximum current. We set $N$, the number of wires such that $I_{0}=I_{\max } / N$, the specified amount of current determined by the engineering constraints. Then the the authors can plot sets of contours or equivalently streamlines on the surfaces, given by $S=k I_{0}, k=1,2, \ldots, N$ and put the wires carrying the currents at points midway between the streamlines.

Note that, for the tilted endcaps, the contours of the stream function on a perpendicular plane to the cylinder axis will always be the same, irrespective of the tilt angle. Two streamlines on the tilted caps, having specified values of the stream function on them will be separated by a distance $1 / \sin (\alpha)$ times larger than the distance between 
similar streamlines on the perpendicular caps. However, at the edge $\left(\rho=R_{S}, z= \pm L_{S}\right)$, distance between the streamlines will be same (be it on the tilted or the perpendicular caps), and hence the same wire connections can be used at the edge coming from the cylindrical surface, for any tilted endcaps.

\section{RESULTS AND DISCUSSIONS}

The authors have used the stream function method for determining the discretized surface currents on the secondary surface using the continuous current density of AlQuaderi et al. (2010). The stream function method guarantees that the current density be continuous and provides a practical way of discretizing it. In the previous work of Baig et al. (2007) stream functions for the cylindrical as well as the caps were found separately. Their stream functions had closed loops on the cylindrical and endcap surfaces separately and current continuity between the cylindrical part and the caps were maintained by hand by chopping off parts of the cylinder and of the caps, to match the contours. This limited the number of loops on the endcaps as there was no guarantee that all the loops (on the cylinder and the caps) would match at the common edge. Compared to their method, our discretized currents are ensured to be continuous on the whole secondary, by virtue of the continuity of the current density. This provides the freedom to choose $I_{0}$ and hence $N$ at The authors' convenience, meeting the engineering constraints.

The authors have solved for the stream function in the most general form since the expressions found can be applied to solely cylindrical surfaces or solely on the endcaps. Both of these have been solved previously in the literature (Wentao et al. (2007), Baig et. al. (2007)) but not in the general form as ours and has not been done previously. The angle $\alpha$ can be varied in our geometry to include the previous work of Baig. et al. (2007) (for $\alpha=90^{\circ}$ ) hence ours is a more general setup since we have more freedom over the optimization procedure having an extra parameter. The values of coefficients in the Fourier series expansions can be found by minimizing the energy functional and the deviation from required values of the magnetic field inside the region of interest and outside the MRI machine (shielding the $B$-filed).

\section{REFERENCES}

Al-Quaderi, G. D., M. Arshad Momen and Tanvir Noor Baig. 2010. Active Endcap Transverse Gradient Coil Design for Magnetic Resonance Imaging: Solving the Current Continuity Issue, Accepted for publication in The Bangladesh Journal of Physics.

Baig, T. N., Timothy P. Eagan, Labros S. Petropoulos, T. K. Kidane, Willam A. Edelstein and Robert W. Brown. 2007. Gradient coil with active endcap shielding. Concepts in Magnetic Resonance Part B, Magnetic Resonance Engineering, 31B(1): 1223.

Michael A. Brideson, K. Larry, Forbes and Stuart Crozier. 2002. "Determining Complicated Winding Pattern for Shim Coils Using Stream Functions and the Target-Field Method”, Concepts in Magnetic Resonance, 14(1): 918. 
Peeren, G. 2003. Stream Function Approach for Determining Optimal Surface Currents”, Ph. D. Thesis, Technische Universiteit Eindhoven.

Shvartsman, SH., M. Morich, G. Demeester and Z. Zhai. 2005. Ultrashort shielded gradient coil design with 3D geometry, Concepts in Magnetic Resonance Part B (Magnetic Resonance Engineering). 26B(1): 115.

Wentao Liu, Donglin Zu, Xin Tang and Hua Guo. 2007. Target-field method for MRI biplanar gradient coil design, J. Phys. D: Appl. Phys. 40: 4418.

(Received revised manuscript on 18 April, 2010) 